\title{
Amantadine Therapy for Fatigue in Multiple Sclerosis
}

\author{
T.J. Murray
}

\begin{abstract}
We carried out a double blind control study of fatigue in 32 patients with multiple sclerosis, comparing amantadine hydrochloride $100 \mathrm{mg}$ twice a day and placebo. On amantadine $31 \%$ had marked improvement; $15.6 \%$ moderate improvement; $15.6 \%$ mild improvement; and $36.5 \%$ unchanged. On placebo, none noted marked improvement; one claimed moderate improvement on either amantadine or placebo. $18.7 \%$ reported mild improvement on placebo: and most of them had similar or more response to amantadine. No patient selected placebo over amantadine at the end of the trial. Overall improvement was seen in $62.5 \%$ of patients on amantadine and $21.8 \%$ on placebo. Additional experience up to two years suggests continued benefit but common and important side-effects.
\end{abstract}

RÉSUMÉ: Traitement de la fatigue par l'Amantadine dans la sclérose en plaques Nous avons étudié par méthode à double-insu chez 32 patients souffrant de sclérose en plaques l'effet comparatif sur la fatigue de l'Amantadine hydrochloré $100 \mathrm{mg}$ deux fois par jour ou du placebo. Avec l'Amantadine, une amélioration marquée de la fatigue fut observée chez $31 \%$ des sujets; chez $15.6 \%$, une amélioration modérée; $15.6 \%$, une amélioration légère. Chez $36.5 \%$ des sujets il n'y eut aucun changement. Avec le placebo, il n'y eut pas d'amélioration marquée. Un patient eut une amélioration modérée avec l'Amantadine ou le placebo; $18.7 \%$ des sujets ont eu une amélioration légère au placebo. Dans presque tous les cas, le résultat avec l'Amantadine fut semblable à celui avec le placebo, ou meilleur. Aucun patient, à la fin de l'essai, choisit le placebo plutôt que l'Amantadine. En résumé, il y eut amélioration chez $62.5 \%$ des patients avec l'Amantadine et chez $21.8 \%$ avec le placebo. Notre expérience subséquente sur une période allant jusqu'à deux ans suggère un bénéfice continu, mais des effets secondaires fréquents et importants.

Can. J. Neurol. Sci. 1985: 12:251-254

Although fatigue is one of the most common and distressing symptoms of multiple sclerosis (MS), there have been few specific references to this problem ${ }^{1,2,3}$ and no specific treatments suggested. In the last four years we have attempted to characterize the fatigue in MS and to evaluate various drug therapies.

\section{Fatigue in Multiple Sclerosis}

Although $90 \%$ of patients with multiple sclerosis complain of fatigue, the data on all cases at the Dalhousie M.S. Research Unit study indicated that $76 \%$ of patients felt the degree and type of fatigue was different from what they experienced prior to the disease. $70 \%$ of the patients noted fatigue during only part of the day, often in the mid-afternoon, but $30 \%$ had it all day, and $27 \%$ had it as a persistent symptom throughout the course of their disease. (Table 1). When patients were asked to list their major symptoms of multiple sclerosis, $40 \%$ listed fatigue as the most serious.

In $33 \%$ of patients the fatigue was aggravated by physical activity and $32 \%$ noted aggravation by emotional stress. $64 \%$ thought that rest improved the fatigue. We noted a small number of patients who complain of a myasthenic-like weakness. Some thought they became weaker after even minor physical activity, and were relieved by brief rest.

\section{Table 1: Fatigue in M.S. Results of Questionnaire Study}

Patients with fatigue

Patients with abnormal fatigue

Present part of the day

Present all day

Present throughout disease course 27\%

Fatigue as the major complaint $40 \%$

Aggravated by physical activity

Aggravated by psychological stress

Relief with rest

Marked

Moderate

Fair or poor

$31 \%$

$33 \%$

$31 \%$

Drug Studies on Fatigue

From the group of patients who complained of persistent fatigue lasting more than three months we obtained cooperation and consent for a series of studies. In only a few instances were the same patients used in more than one study. All patients were clinically definite by the Schumacher criteria. Ten cases were selected initially for an open preliminary trial on each drug to see if there was enough positive response to warrant a larger double blind control study. Patients were instructed to describe 
their level of fatigue as none, mild, moderate or severe in a diary three times a day, and to summarize. Aspects of depression were evaluated in the patients, and those with any evidence of clinical depression were excluded from the trials.

Over a period of four years we evaluated four drugs. Methylphenidate was selected as a mild CNS stimulant. Pyridostigmine bromide was used in the subgroup of patients that had myasthenic-like weakness and fatigue. Baclofen was studied because some patients treated for flexor spasms and spasticity said they felt better generally. Amantadine hydrochloride was studied because of the serendipitous observation that one of our patients had a remarkable improvement in fatigue while taking amantadine to prevent influenza.

\section{RESULTS}

A preliminary open trial on ten M.S. patients with fatigue using methylphenidate gave no positive responses, and none of the patients wished to continue the drug following a three month trial. In the study of ten patients treated with pyridostigmine bromide, no patients improved and none of the patients wished to continue the drug at the end of the trial. In the trial of ten patients using baclofen we found no appreciable affect on fatigue, five complained of excessive weakness, and two had improvement of periodic muscle spasms. None of the patients in these three trials wished to continue on the drugs for the relief of fatigue following the preliminary study.

We gave amantadine to eighteen patients in a preliminary open trial and fourteen had a positive response. We therefore designed a double-blind crossover study in forty patients. Forty patients were selected for a double-blind crossover study over six weeks with a one week washout period between active drug and placebo. All forty patients had a complaint of fatigue which they felt was abnormal, or greater than normal for more than three months and in most the symptom had been present for years. The patients ranged from 0 (normal) to 7 (wheelchair) on the Kurtzke Disability Scale. Most patients were in the 0 to 3 disability scale, as mobile and working patients were often the most disturbed by the effects of constant fatigue.

Half of the patients were given amantadine $100 \mathrm{mg}$ twice a day, and half on placebo, similar in appearance and taste. Eight patients declined to enter the study before the drug was initiated, so the trial was initiated and completed on thirty-two patients. (Table 2). Evaluation, explanation and reassessments were carried out by the neurologist, fatigue evaluation by a research assistant and by daily patient diaries. Medication and placebo were administered by a pharmacist.

On amantadine $31 \%$ ( 10 cases) noted a marked improvement; usually indicating that they now felt normal. $15.6 \%$ ( 5 cases) felt that the improvement was moderate, and $15.6 \%$ (5 cases) felt that it was mild. $36.5 \%$ ( 12 cases) felt that there was no appreciable change. $25 \%$ ( 8 cases) reported side effects on amantadine, and one patient later discontinued the drug because of nausea and hallucinations.

On the placebo no patients claimed marked improvement, one felt that there was moderate improvement but that patient also claimed moderate improvement for amantadine. $18.7 \%$ (6 cases) claimed mild improvement on placebo, but these same patients all noted mild, moderate or marked improvement on amantadine as well. No patient felt that the placebo was more effective than amantadine, although two amantadine responders preferred the placebo because they had side effects on amantadine. $18.7 \%$ ( 6 cases) complained of side effects on the placebo and one patient discontinued the drug as she felt it was making her spasticity worse. In general, the placebo side effects were milder than those recorded on amantadine.

At the end of the six week trial the patients were asked to make a selection of Drug A, Drug B or No Drug for continuing therapy. Nineteen patients selected amantadine. No patient selected the placebo. Thirteen patients did not wish to continue on the drug. Seven of the patients who responded well to amantadine did not wish to continue on the drug because of only mild to moderate improvement, expense of the drug, or side effects. It is interesting to note that four of these patients have subsequently returned to our Clinic and requested amantadine, so the eventual results were 23 on long term amantadine, 0 on placebo and 9 on no drug.

Side effects of amantadine included hallucinations, nausea, gastric irritation, early morning wakening and hyperactivity. Three patients complained of hyperactivity, and they did not like the feeling, but continued the drug. One patient had a flu-like illness while on amantadine. A total of 7 out of 32 cases reported side effects on amantadine. On the placebo 6 out of 32 cases reported side effects, including nausea, drowsiness, malaise, and increased spasticity.

In a short term trial change in disability scale is not expected, although we carried out such assessments. A few patients showed changes in their examination but this would be expected in any group study of MS. A number showed improvement in mobility and function because of marked change in their energy but not because of altered motor dysfunction.

\section{DISCUSSION}

These studies suggest that there are four categories of fatigue in multiple sclerosis: (1) normal fatigue; (2) episodic fatigue, (3) continuous fatigue; and (4) myasthenic-like fatigue.

In some patients the fatigue was the first symptom of multiple sclerosis, preceding other specific neurological symptoms by months and occasionally years. In some patients it is present

Table 2: Double Blind Crossover Study (32 Cases)

\begin{tabular}{|c|c|c|c|c|c|}
\hline \multicolumn{6}{|c|}{ Improvement } \\
\hline & Marked & Moderate & Mild & No change & Overall improvement \\
\hline Amantadine & $10(31 \%)$ & $5(15.6 \%)$ & $5(15.6 \%)$ & $12(37.5 \%)$ & $20(62.5 \%)^{* *}$ \\
\hline Placebo & 0 & $1(3 \%)$ & $6(18.7 \%)^{*}$ & $25(78 \%)$ & $7^{*}(21.8 \%)$ \\
\hline
\end{tabular}


periodically, lasting for a number of weeks, and then disappearing, but in other patients it has been present throughout the duration of their disease. The episodic occurrence is sometimes dramatic with marked fatigue for two to four weeks which then clears, often without any other evidence of neurological disease. Fatigue may also worsen as the disease worsens, and worsen when attacks occur. In other patients there appears to be no relationship with the activity of their disease, and many of our patients with marked fatigue have been in remission for a number of years.

We have demonstrated that fatigue is present in most patients with multiple sclerosis, but it appears to be an abnormal and excessive fatigue in $76 \%$ of patients. Despite many other problems and disabilities, $40 \%$ of MS patients complained that fatigue is their major and most distressing symptom. Indeed, to many experienced clinicians who deal with large numbers of multiple sclerosis patients, this unusual fatigue is felt to be almost diagnostic of multiple sclerosis. Freal et $\mathrm{al}^{3}$ recently reported fatigue in $78 \%$ of MS patients and indicate an unpublished study showing $90 \%$ with fatigue.

Amantadine hydrochloride is an antiviral agent which acts by inhibiting penetration of the virus into the host cell. It does not appear to be virucidal, and does not suppress antibody response. It may augment dopaminergic activity and is thus a useful secondary drug in Parkinson's disease ${ }^{4}$. It has also been reported to be useful in chorea ${ }^{5}$, manganese poisoning ${ }^{6}$, panencephalitis ${ }^{7}$, drug induced parkinsonism ${ }^{8}$, depression ${ }^{9}$, dementia ${ }^{10}$, Down syndrome $^{11}$ and essential tremor ${ }^{12}$. Many of these reports are difficult to evaluate, but it emphasizes the central nervous system effects of this interesting drug.

There are anecdotal reports of MS patients improving in function and disability status on amantadine ${ }^{13.14}$ but a well controlled study showed no significant improvement with this therapy ${ }^{15}$. No specific mention is made of fatigue in these reports, but in our study it appears the only evident improvement is in fatigue but this improvement in energy may secondarily improve function.

Two major and obvious questions remain unanswered. What is the origin of this unusual fatigue that seems unique to multiple sclerosis? It is not a fatigue that we tend to see in a majority of patients with other disabling neurological diseases, such as amyotrophic lateral sclerosis, cerebral palsy or traumatic damage to the nervous system. Second, what is the explanation of the pharmacological effect of amantadine? It clearly is not due to its antiviral potential. Patients note a response to the drug within 24 hours, and on stopping, a return of the fatigue within twentyfour hours, suggesting a chemical response. It may be due to its dopaminergic effects, and we are planning some trials with other dopaminergic and amphetamine drugs.

Although our data are incomplete on long term use of this therapy, we are in the midst of a long-term trial on 100 patients and our initial results suggest that the effect of amantadine is long-lasting, unlike the waning affects in Parkinson's disease but associated with significant side-effects. We now have patients who have been taking amantadine hydrochloride for over two years, and the improvement continues in those who initially responded. We have not been able to predict those who will respond best, although further studies are planned. We are now developing electrophysiological measures to try to introduce an objective assessment to what is essentially a subjective phenomenon.
Table 3: Side-Effects

\section{Amantadine ( $7 / 32$ cases)}

hallucinations, nausea*

gastric irritation

nausea

early morning wakening

hyperactivity (2)

flu-like illness

* resulted in cessation of drug
Placebo (6/32 cases)

increased spasticity*

nausea

drowsiness

malaise

malaise*

nausea

\section{CONCLUSIONS}

1. Fatigue occurs in most patients with multiple sclerosis, but appears to be a specific and abnormal fatigue in $76 \%$. In $40 \%$ of patients in the Dalhousie MS Research Unit it is their most bothersome symptom.

2. In preliminary trials with CNS stimulants (methylphenidate), anticholinergic drugs (pyrostigmine bromide) and antispasticity agents (baclofen), patients reported no relief of fatigue.

3. Amantadine hydrochloride gave a mild to marked response in $77 \%$ of the patients in a preliminary trial, and $62.5 \%$ in a double-blind crossover study compared to $21.8 \%$ receiving a placebo. Side-effects occurred in $25 \%$.

4. Initial experience with long term therapy up to two years suggests a continuing effectiveness of this therapy in cases who initially respond but with side-effects in most patients.

\section{ACKNOWLEDGEMENTS}

The author wishes to thank Dr. Alexander Cameron who told us about the effect of amantadine in a patient with M.S. We are grateful to DuPont Canada Inc. for assistance; to Pauline Weldon, Coordinator of the Dalhousie M.S. Research Unit; Jean MacLean for secretarial assistance; Margaret Liston for research assistance; and to the patients of the Dalhousie M.S. Research Unit for their cooperation and understanding.

\section{REFERENCES}

1. MacAlpine D, Lumsden CE, Acheson ED. Multiple Sclerosis: A reappraisal. Edinburgh: Churchill Livingston 1972; 115-116.

2. Herndon RM, Rudick R. Fatigue in M.S. M.S. Quarterly. Summer $1981 ; 4-14$.

3. Sandy KR. Anergia and depression as early features of Multiple Sclerosis. S Afr Med J 1983; 732.

4. Schwab RS, England AC, Poskanzer DC, Young RR. Amantadine in the treatment of Parkinson's Disease. JAMA 1969: 208:1168.

5. Gray MW, Herzberg L, Lenman JAR, et al. Anantadine in chorea. Lancet 1975; 2:132-133.

6. Schunk W. Manganese poisoning. Dsch Gesundh Wes 1976; $31: 1847$.

7. Haslam RHA, McQuillen MP, Clark DB. Amantadine Therapy in subacute sclerosing panencephalitis. Neurology 1969; 19:1080.

8. Pacifici GM, Nardini M, Ferrari $P$, et al. Effect of Amantadine on drug-induced Parkinsonism: relationship between plasma levels and effect. Br J Clin Pharmac 1976: 3:883.

9. Rizzo M, Biandrate $P$, Tognoni G, et al. Amantadine in depression: relationship between behavioral effects and plasma levels. Europ J Clin Pharmacol 1973; 5:226-228.

10. Chierichetti SM, Ferrari $P$, Sala $P$, et al. Effects of Amantadine on mental status of elderly patients. Curr Ther Rs 1977; 22:158-165.

11. White G. Improving babies with down's syndrome. Med J Australia $1974 ; 2: 184$

12. Critchley E. Clinical Manifestations of Essential Tremor. J Neuro Neurosurg Psychiat 1972; 35:365-372. 
13. MacDonald JR. Empirical use of amantadine in Multiple Sclerosis. Wink CAS (ed) Symmetrel in Virology. Geigy Pharm Macdesfield p 48-50.

14. Schapira M. Amantadine in the treatment of Multiple Sclerosis. J Roy Coll Gen Prac 1974; 2:411-413.

15. Ellison GW, Mickey MR, Myers LW, et al. A Pilot study of Amantadine treatment of Multiple Sclerosis. In: Progress in Multiple
Sclerosis Research. Bauer HF, Doser S, Ritter G, (eds) Berlin: Springer - Verlag 1980; 407.

16. Freal JE, Kraft GH, Coryell JK. Symptomatic Fatigue in Multiple Sclerosis. Arch Phys Med Rehabil 1984; 65:135-138. 